\title{
On Large Eddy Simulation Based Conjugate Heat Transfer Procedure for Transient Natural Convection
}

\author{
M. Fadl ${ }^{(1)}$ \\ Department of Engineering Science \\ University of Oxford, Oxford OX1 3PJ, UK,
}

\section{L. $\mathrm{He}^{(2)}$}

Department of Engineering Science

University of Oxford, Oxford OX1 3PJ, UK

email: Li.He@eng.ox.ac.uk

(1, currently: CREST, Loughborough University, email: m.s.fadl@lboro.ac.uk)

(2, corresponding author, email: Li.He@eng.ox.ac.uk) 


\section{ABSTRACT}

Natural convection is an important heat transfer mode for flexible operations of gas turbines and steam turbines. Its prediction presents considerable challenges. The strong inter-dependence between fluid and solid parts points to the need for coupled fluid-solid conjugate heat transfer (CHT) methods. The fundamental fluid-solid time scale disparity is further compounded by the long-time scales of practical turbine flexible operations. In addition, if a high-fidelity flow model (e.g. Large Eddy Simulation, LES) is adopted to resolve turbulence fluctuations, extra mesh dependency on solid domain mesh may arise.

In this work, understanding of the extra solid mesh dependency in a directly coupled LES based CHT procedure is gained by an interface response analysis, leading to a simple and clear characterization of erroneously predicted unsteady interface temperatures and heat fluxes. A loosely coupled unsteady CHT procedure based on a multi-scale methodology for solving problems with large time scale disparity is subsequently developed. A particular emphasis of this work is on efficient and accurate transient CHT solutions in conjunction with the turbulence eddy resolved modelling (LES) for natural convection. A two-scale flow decomposition associated with a corresponding time step split is adopted. The resultant triple-timing formation of the flow equations can be solved efficiently for the fluid-solid coupled system with disparate time scales. The problem statement, analysis and the solution methods will be presented with case studies to underline the issues of interest and to demonstrate the validity and effectiveness of the proposed methodology and implemented procedure.

\section{INTRODUCTION}

Thermal analysis is necessarily required as part of a turbomachinery design system. It is normally the case that design methods and tools are much more well-established for nominal steady (or periodically unsteady) states than those for dynamic transient processes. Increasingly, adequate predictions of transient thermal characteristics during start-up, shut-down, load changing conditions of power generation operations for steam turbines, gas turbines and aero-engines are of great interest. For example, the growing renewable energy resources in market share require more flexible steam turbine operations. In order to design reliably a steam turbine for flexible operations, designers need to understand and predict dynamic thermal characteristics of steam turbine components during a transient process. Similarly, for gas turbines, the thermal fatigue life span is also strongly dependent on the predictability of transient thermal behaviour of the hot components during an operation cycle. 
Turbine dynamic thermal characteristics are strongly influenced by various convective heat transfer phenomena. Analysis and prediction of the problems related to natural convection are more challenging with less well-established theories, methods, or even empirical correlations, compared to the forced convection counterparts. However natural convection can be dominantly influential for a large part of a flexible operation process. This important practical requirement has led to some active developments of physical modelling based methods. A notable recent example is the development of a new overconductivity based method, backed up by experimental measurements for assessing dynamic thermal characteristics during steam turbine shutdown and cooling processes [1], [2].

In terms of higher fidelity computational fluid dynamics (CFD) based heat transfer methods, most widely used procedures for convective heat transfer tend to be based on the heat transfer coefficient (HTC) as the main boundary condition for a solid conduction solution. The HTC based methods would work well if HTC is predominantly determined by the flow, largely (if not completely) independent of the wall temperature, as commonly assumed. This conventional wisdom may be in question if the flow near a solid surface is influenced by the wall temperature in a non-negligible manner, e.g. as indicated for gas turbine blades subject to forced convection heat transfer with a large enough temperature difference between the wall and main flow [3], [4] and [5].

For a natural convection problem, the near-wall flow will be strongly influenced by the wall temperature, which in turn affects the convective heat transfer, thus the wall temperature. This strong inter-dependence between the fluid and solid domains would cause greater difficulties for HTC based methods for natural convection. Consequently, for natural convection, we would be more inclined to consider developing and applying a coupled convection-conduction conjugate heat-transfer (CHT) approach, e.g. [6] and [7]. Furthermore, the lack of well-established general theoretical and empirical modelling methods would also tend to ask for higher flow model fidelities for natural convection, and in the case of a turbulent flow, LES would be a natural candidate for flow modelling if feasible.

Given the need for a LES based CHT method for transient natural convection analysis for the problems of interest, what are options available or potentially available?

A numerically straightforward option may be the direct coupled unsteady CHT, where both the fluid and solid domains are integrated in time simultaneously. The main issue common to all CHT methods is the disparity in time scales between fluid and solid. For a turbine forced convection problem, the relative time-scales for the two domains can differ by a factor of $10^{4}$ [8]. Fully coupled unsteady CHT simulations 
using the same discretization in space and time for both fluid and solid domains can be prohibitively expensive when a practical application such as a turbine flexible operation is to be dealt with. The problem would be difficult for unsteady Reynolds-averaged Navier-Stokes (URANS) based CHT methods, but much worse for those based on LES solvers in which smaller time steps by one or two orders of magnitude would be required.

There is also another aspect, which has been rarely addressed, regarding the solution consistency and accuracy of a directly coupled CHT method with LES. For a LES CHT solution of a turbine forced convection problem, predicted unsteady heat flux fluctuations can be remarkably sensitive to the fluidsolid interface treatment, as shown by He [9]. In a combustor instability analysis using LES, a considerable mesh dependence of the solid domain is observed by Shahi et al [10]. Qualitatively this may be attributable to a short penetration depth of a high frequency disturbance relative to mesh resolution. However, understanding and quantification of the behaviour are still lacking for the transient CHT modelling and method development.

Loosely coupled CHT methods, in which the fluid and solid domains are solved by separate solvers and coupled together at an interface iteratively, have been more commonly adopted. Furthermore, the time scale disparity between the fluid and solid domains provides a common justification for a quasi-steady transient CHT approach, i.e. coupling a steady flow solver quasi-steadily with an unsteady solid conduction solver. The quasi-steady approach has been widely adopted recently with different flow fidelities, from RANS (e.g. [11] and [12]) to LES (e.g. [13] and [14]). Recent efforts also include those on faster and more robust interface treatments (e.g. [15]). However, we note that all previous loosely coupled CHT methods have been developed and applied only to forced convection problems. Very recently, the present authors have also developed a URANS based loosely coupled method for transient natural convection analyses (Fadl et al [16]). But, to the authors' knowledge, there have been no loosely coupled CHT analyses with LES flow models for natural convection.

The present research effort is chiefly motivated to explore the applicability of a LES based loosely coupled CHT method for transient natural convection, relevant to the turbine flexible operations. There are two primary questions in mind:

i) Given that a loosely coupled CHT should be seemingly more computationally efficient than a direct coupled CHT, are there any advantages of using a loosely coupled method in terms of computational accuracy and consistency for natural convection? 
ii) Further, common to previous loosely coupled CHT methods is the quasi-steady assumption. Given this, can we develop a loosely coupled CHT method without this modelling restriction for natural convection, whilst still being computationally as efficient?

The present paper is organized with an intent to clearly identify and understand the issues before addressing them. After introducing the direct CHT method as it is in the commercial code FLUENT, we present computational studies to highlight the issue of the direct coupling when applied to natural convection with LES. Then, a new multi-scale loosely coupled CHT methodology as implemented in FLUENT is described. The computational accuracy and consistency of interest are examined by contrasting the loosely coupled solutions with the directly coupled CHT solutions with the same LES flow solver. The influence of a quasi-steady model will also be assessed to underline the advantages of the present multi-scale loosely coupled procedure.

\section{DIRECT CHT METHOD AND ANALYSIS}

\subsection{Baseline Flow Solver for Direct Coupled CHT}

The flow is governed by the 3D unsteady compressible flow equations, in a short form:

$$
\frac{\partial U}{\partial t}+R_{f}(U)=0
$$

where $U$ is the vector of conservative variables and $R_{f}$ is the standard flux residuals as for typical forced convection computations. In the present studies for natural convection, we adopt the full buoyancy term in FLUENT, due to gravitational acceleration directly proportional to fluid density, in line with the fully compressible flow model as adopted here.

For turbulent flows at high Rayleigh number conditions, we use a LES solver. The standard Smagorinsky sub-grid model is adopted. Effectively unsteady flow equations will be integrated numerically in time. A pseudo time $\tau$ can be introduced for local sub-iterations by time-marching at each physical step. A dual-timing form with an implicit source term treatment should be in a form (for a physical time step $\mathrm{N}$ ):

$$
\left(\frac{\partial \mathbf{U}}{\partial \tau}\right)^{N, k}+\frac{1.5 \mathbf{U}^{N, k}-2 \mathbf{U}^{N-1}+0.5 U^{N-2}}{\Delta t}+R_{f}\left(\mathbf{U}^{N, k-1}\right)=0
$$


where $\mathrm{k}$ is the index of the sub-iterations. When the sub-iterations converge, the pseudo time term vanishes, and the full 2nd order temporal approximation of the unsteady flow equation (Eq.1) fully recovers.

For a solid domain, the temperature field is governed by the unsteady conduction equation.

$$
\frac{\partial T}{\partial t}+R_{S}(T)=0
$$

The semi-discrete unsteady conduction equation in the dual-timing form for time step $\mathrm{N}$ and subiteration $\mathrm{k}$ is:

$$
\left(\frac{\partial T}{\partial \tau}\right)^{N, k}+\frac{1.5 T^{N, k}-2 T^{N-1}+0.5 T^{N-2}}{\Delta t}+R_{S}\left(T^{N, k-1}\right)=0
$$

When a directly coupled CHT problem is solved, the physical conditions of temperature and heat flux continuity, Perelmans [17] between the fluid side (f) and solid side (s) are:

$$
\begin{aligned}
& q_{S}\left(T_{S}\right)^{N, k}=q_{f}\left(T_{f}\right)^{N, k} \\
& T_{S}^{N, k}=T_{f}^{N, k}
\end{aligned}
$$

A discretized form of Eq.(5a), by means of the temperature continuity across the wall surface, leads to an explicit expression of the wall temperature enabling both sides to be marched forward in time concurrently.

\subsection{Computational Set-up}

A simple configuration is taken for the present studies, though the dimensions adopted are intended to be relevant to the turbine flexible operations.

The fluid domain has an outer diameter of $1.5 \mathrm{~m}$ and an inner diameter of $0.5 \mathrm{~m}$. The solid domain has an outer diameter of $2.0 \mathrm{~m}$, and its inner boundary is the interface with the fluid domain. The inner wall of the fluid domain and the outer wall of the solid domain are subject to isothermal temperature conditions $\left(\mathrm{T}_{\mathrm{i}}=800 \mathrm{~K}, \mathrm{~T}_{\mathrm{o}}=400 \mathrm{~K}\right)$. For resolving turbulent eddies, a 3D domain is needed. In this case, the domains have a width of $0.1 \mathrm{~m}$ in the axial-direction, and the adiabatic viscous wall conditions are applied at the two side surfaces.

The nominal fluid properties can be calculated at the mean temperature of $600 \mathrm{~K}$. The values of fluid dynamics viscosity, thermal conductivity and specific heat are 3.017 x10 $10^{-5} \mathrm{~kg} / \mathrm{m} \mathrm{s}, 0.04661 \mathrm{~W} / \mathrm{m} \mathrm{K}, 1.0511$ 
$\mathrm{kJ} / \mathrm{kg} \mathrm{K}$, respectively. For this internal natural convection in an annulus, a Rayleigh number based on the gap-width is $2.64 \times 10^{7}$.

For the solid domain, it is assumed that the properties of the solid are constant and independent of temperatures. Steel is taken as the material with the following properties: heat capacity of $502.48 \mathrm{~J} / \mathrm{kg} \mathrm{K}$, density of $8030 \mathrm{~kg} / \mathrm{m}^{3}$ and thermal conductivity of $16.27 \mathrm{~W} / \mathrm{m} \mathrm{K}$.
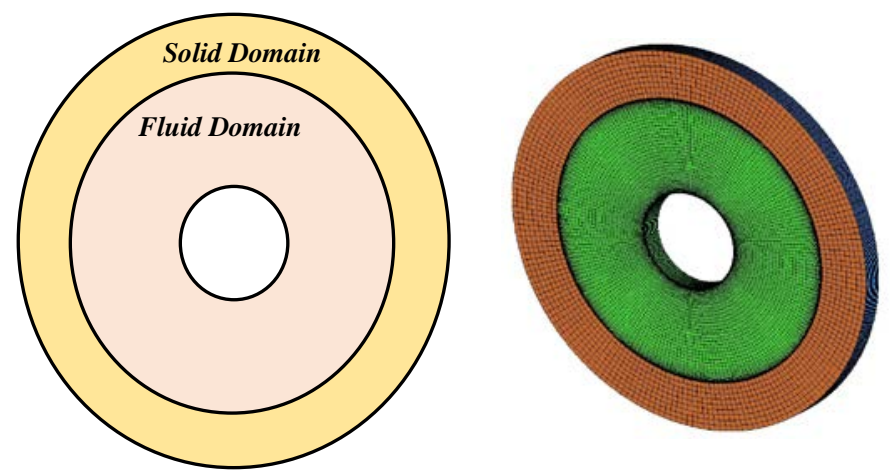

Fig. 1, Computational Domain and mesh (CHT).

A structured mesh system from Ansys ICEM is adopted for both domains as shown in Fig. 1. The pressure-based solver is selected for all fluid solutions. The temporal discretization is the $2^{\text {nd }}$ order implicit one. Typically, 20 sub-iterations are taken for each physical time step. All LES solutions are initialized from steady RANS solutions with the $k-\varepsilon$ model for turbulence closure.

A typical example of the LES solution of the natural convection problem is shown in Fig. 2 for the time-averaged and instantaneous velocities.

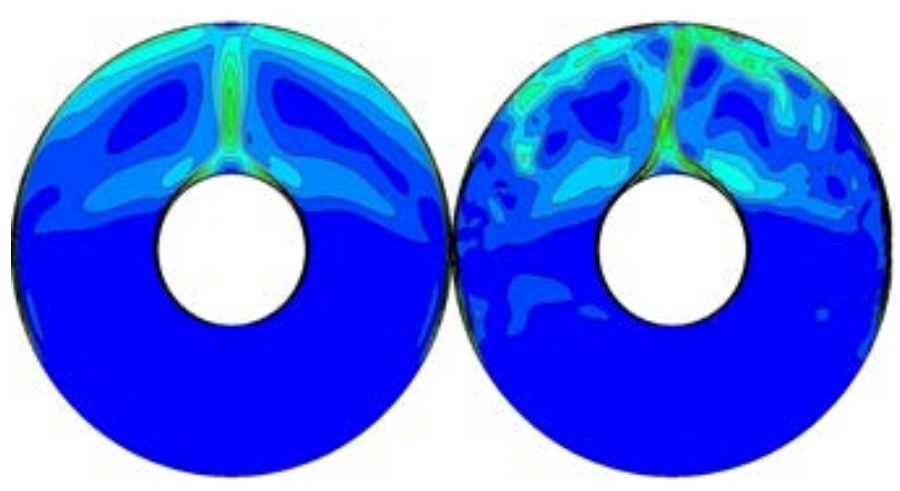

Fig. 2, Time-mean (left) and instantaneous (right) velocity contours (range: $0-0.75 \mathrm{~m} / \mathrm{s}$ ) of LES solution (mid-axial plane). 


\subsection{Sensitivity Studies of Direct Coupled CHT}

This section provides the results as the evidence to underline the problem statement in relation to the extra solid mesh dependency caused by the interface treatment for a LES based CHT solution. We first examine the grid and time step dependency for a fluid domain in isolation with a specified isothermal wall condition. We then look at a steady RANS based CHT case to provide a reference point regarding the mesh dependency for the solid domain. These separate case studies will form a clear contrasting basis for comparisons when a LES-CHT analysis is performed.

\subsubsection{Mesh and Time-step Sensitivity of Fluid Domain}

The initial test is carried out for the fluid domain only with the isothermal wall conditions specified at the inner and outer wall surfaces. The purpose is to establish a reasonably grid-independent mesh regime for LES for the fluid domain. For natural convection problems, the flow is predominantly driven by large scale movement, and appears to be much less sensitive to the near-wall fine mesh resolution, compared to that for forced convection. Two mesh densities are considered for the fluid domain. Mesh 1 has 200000 nodes (50x200x20 for the r, $\theta$ and z directions) with the radial spacing of the first cell of $1 \mathrm{~mm}$. Mesh 2 has 400000 nodes (100x200x20) with the first cell to wall boundary distance of $0.5 \mathrm{~mm}$.

Fig.3 shows the time histories of heat flux at the middle position on the top boundary surface of the fluid domain, where the main upward convected stream impinges on the wall (Fig.2). The corresponding power spectrum is shown in Fig.4. The time traces show very similar fluctuation characteristics in line with the spectra for the two meshes. The time-averaged heat fluxes for the two meshes (indicated in Fig.3) are also in a good agreement. The low-density mesh, though rather coarse for typical LES solutions for convective heat transfer, is thus taken for further analyses.

The next aspect to examine is the time step dependence for the given mesh. Three time steps are considered here, $\Delta \mathrm{t}=0.1 \mathrm{~s}, 0.05 \mathrm{~s}, 0.025 \mathrm{~s}$. The turbulence spectra of the axial velocities from the solutions of the three time steps are compared in Fig.5. Based on the test and the comparison, the time step of $\Delta \mathrm{t}=0.1$ $\mathrm{s}$ is judged to be sufficient and thus taken for further analyses.

For the rest of CHT sensitivity studies, the LES solver for the fluid domain will use the same mesh and the same time step, as identified in this section. 


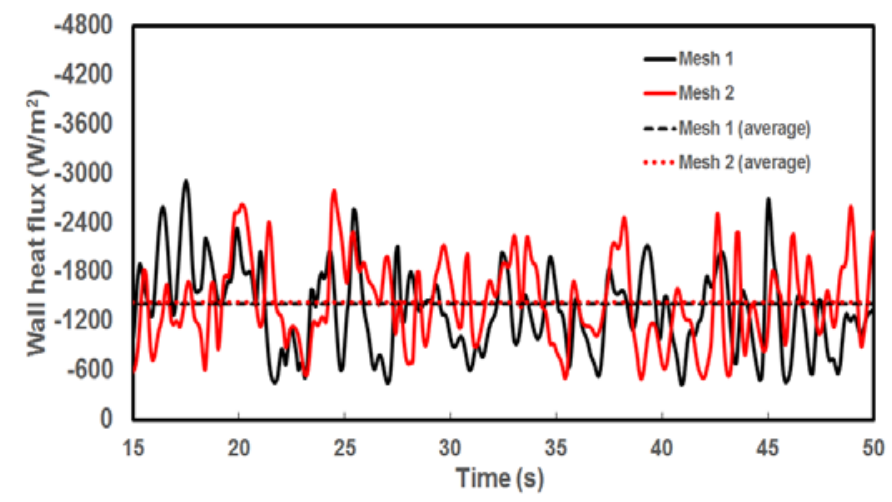

Fig. 3. Time histories of wall heat flux for two fluid meshes (Fluid-domain-only LES).

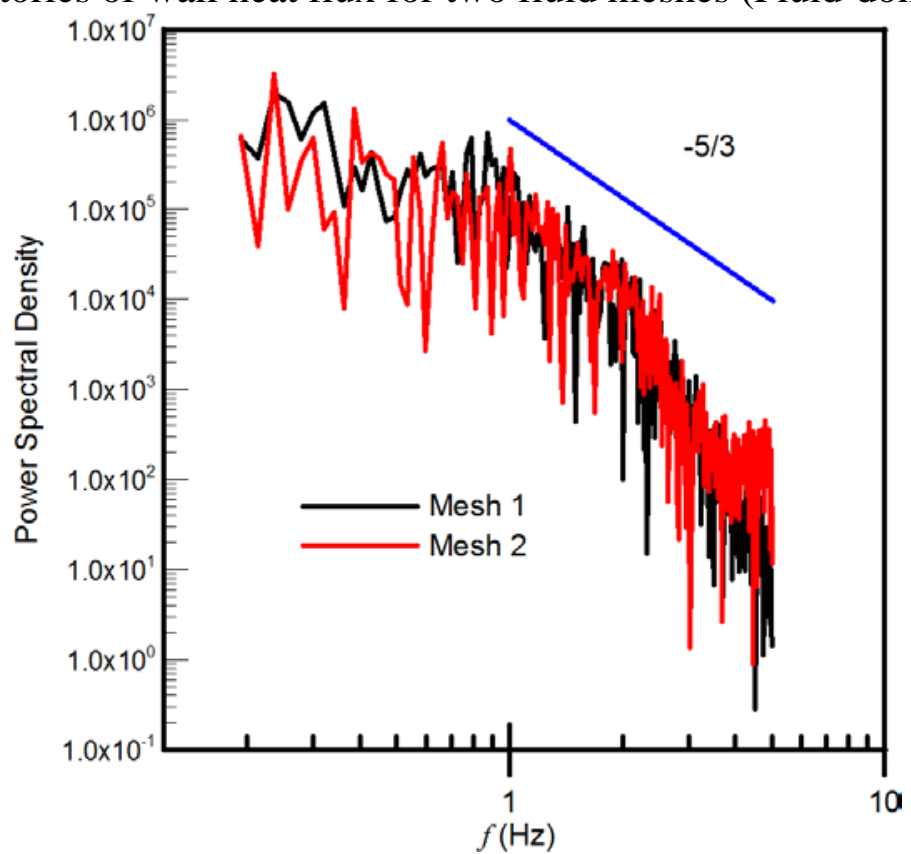

Fig. 4. Turbulence spectra of two different fluid meshes (Fluid-domain-only LES). 


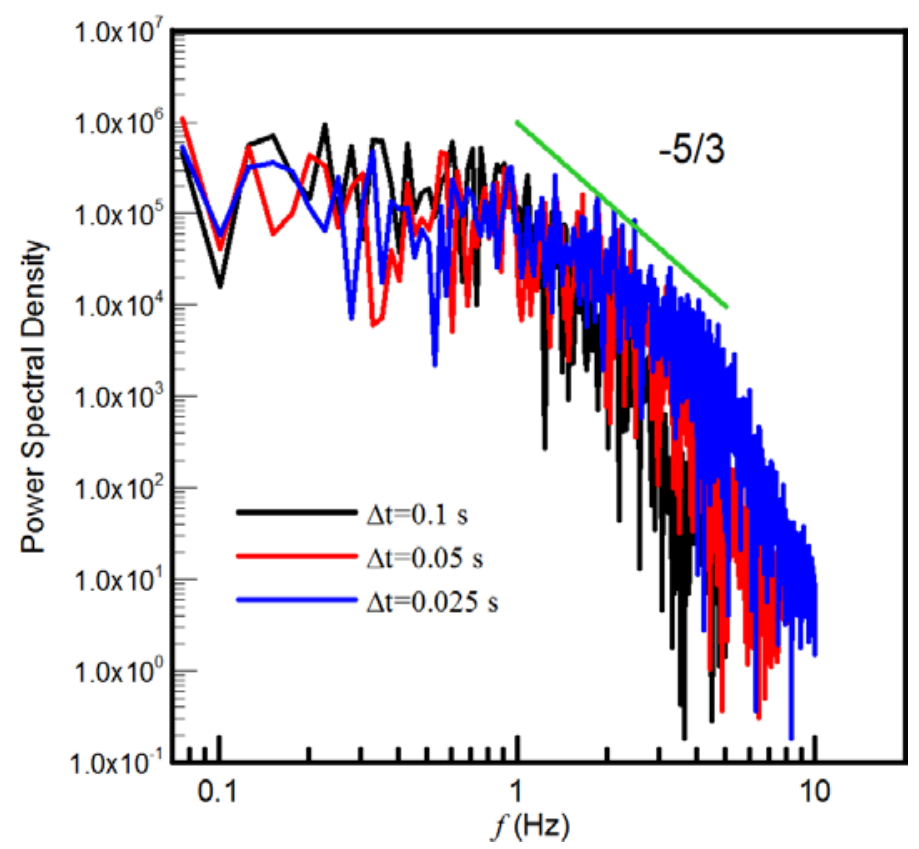

Fig. 5, Turbulence velocity spectra of different meshes (fluid-domain-only LES).

\subsubsection{Solid Domain Mesh Sensitivity (RANS).}

We now turn the attention to the mesh of the solid domain, which is the focal point of the present work. Four meshes are examined as listed in Table 1. For the present interest, the circumferential mesh spacing and number of points are fixed, and we only change the mesh density in the radial direction. Effectively the radial mesh spacing is changed by a factor 8 from the coarsest mesh (Mesh 1) to the finest mesh (Mesh 4).

Table 1: Mesh Densities of Solid Domain

\begin{tabular}{|c|l|l|l|l|}
\hline Solid Mesh & Mesh 1 & Mesh 2 & Mesh 3 & Mesh 4 \\
\hline Number of Nodes & $\mathbf{3 9 2 0 0}$ & $\mathbf{7 8 4 0 0}$ & $\mathbf{1 5 6 8 0 0}$ & $\mathbf{3 1 3 6 0 0}$ \\
\hline $\begin{array}{l}\text { Radial spacing of } \\
\text { the first cell }[\mathrm{m}]\end{array}$ & $\mathbf{0 . 0 2 7 8}$ & $\mathbf{0 . 0 1 3 1 7}$ & $\mathbf{0 . 0 0 6 4 1}$ & $\mathbf{0 . 0 0 3 1 6 7}$ \\
\hline
\end{tabular}

The solid domain mesh sensitivity is examined first by using a steady RANS based CHT solver for natural convection. A grid dependency study in this case would serve to indicate not only the mesh dependency behaviour of the solid domain, but also that of the interface, when not subject to short scale unsteady disturbances. 
Fig. 6 shows the interface temperature contours for the coarsest and finest solid meshes. The differences between the two solutions are also included with the corresponding levels given in the contour legend on the left of the figure. The corresponding comparison of the interface heat fluxes is shown in Fig. 7. We can see that the results from the two meshes are in excellent agreement, with a maximum discrepancy in the order of $0.05 \%-0.1 \%$ for both the temperature and the heat flux. Therefore, for the four solid meshes tested, the steady solid domain solutions exhibit hardly noticeable mesh dependency. A similar behavior of the grid-dependency in relation to the solid mesh can also be said about the interface, when subject to steady CHT simulations.

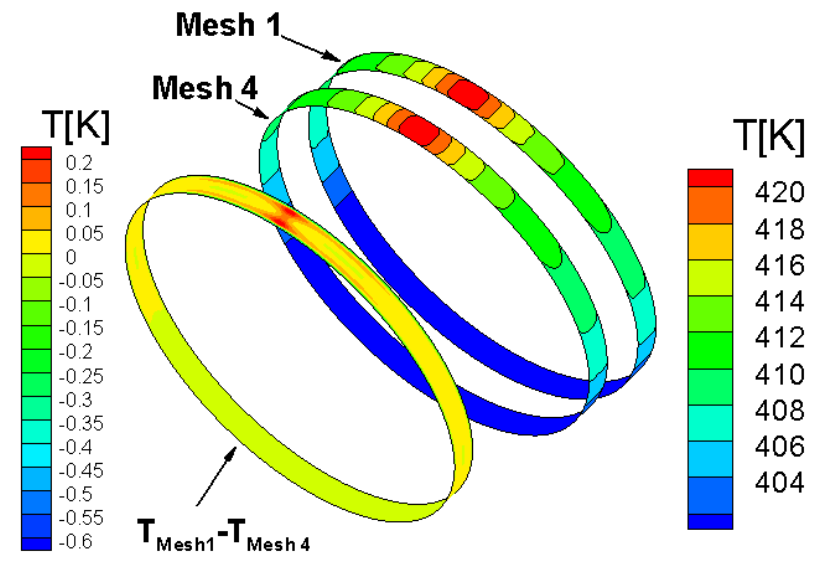

Fig. 6 Interface temperatures for different meshes (Steady RANS based CHT).

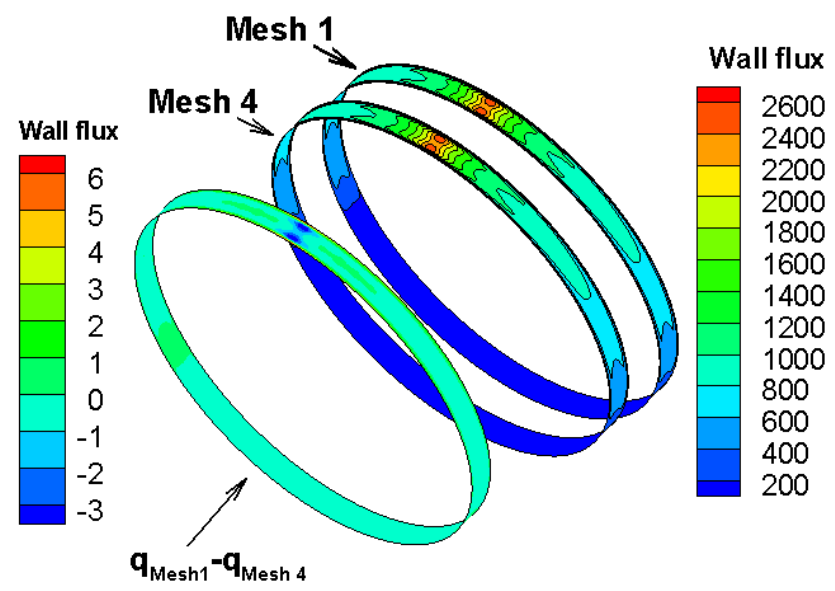

Fig. 7, Interface heat fluxes for different meshes (Steady RANS based CHT). 


\subsubsection{Sensitivity of LES CHT Solution to Solid Domain Mesh}

Having examined the sensitivities for the LES flow solutions for the fluid domain in isolation, for the solid domain solutions as well as the interface as part of the steady CHT solutions, we now focus on the LES as part of the directly coupled CHT solutions.

Firstly, we note a clearly more pronounced mesh dependence of time-averaged interface temperatures as shown Fig.8. Compared to the steady CHT case (Fig. 6), the temperature differences between the twoextreme solid meshes are about 5 times bigger.

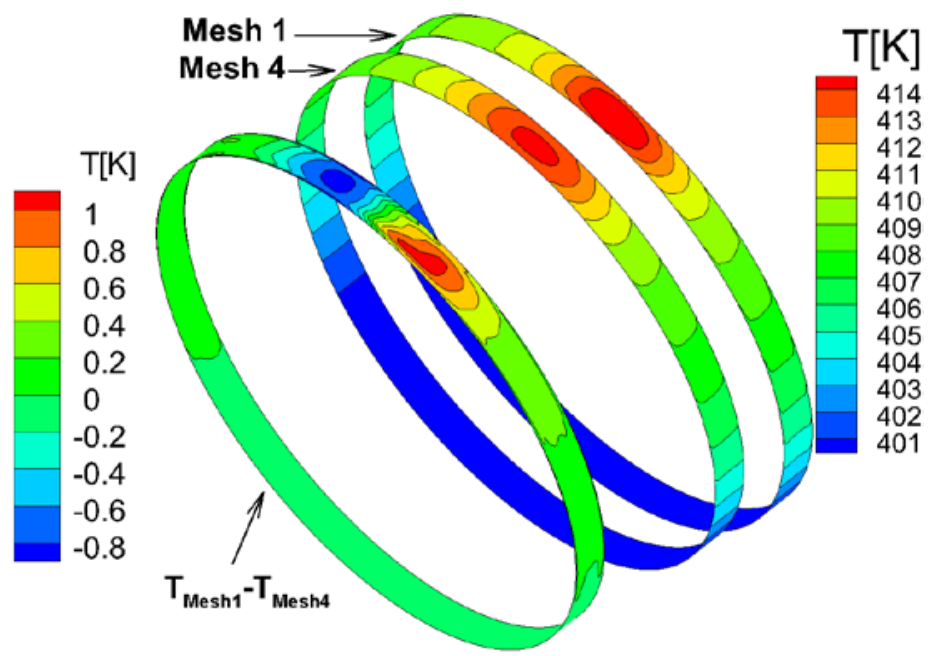

Fig. 8 Interface temperatures for different meshes (Direct coupled CHT using LES).

Notable discrepancies between the two meshes also appear in the time-averaged heat fluxes on the interface as shown in Fig.9. Now the maximum local differences in the time averaged heat fluxes between the two meshes can be as high as $15 \%$. 


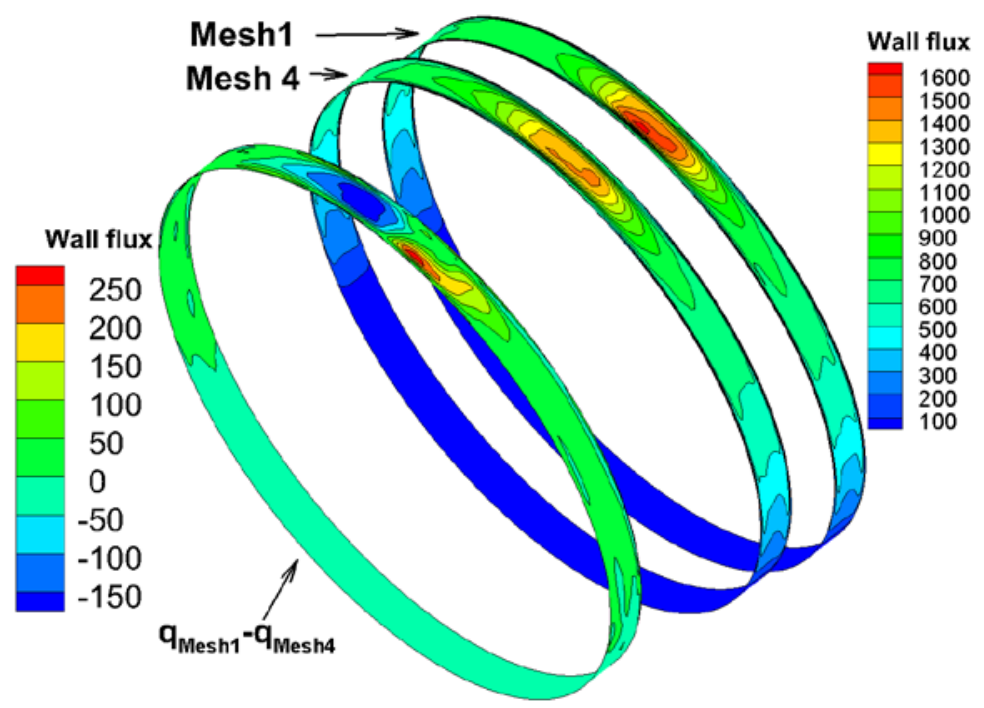

Fig. 9 Interface heat fluxes for different meshes (Direct coupled CHT using LES)

A further indication of the mesh dependence of the direct CHT solutions is illustrated in the time traces of the interface temperatures at the mid-point of the upper surface. The time traces of the wall temperatures for the four solid meshes exhibit markedly different unsteady fluctuations, in addition to the drifting mean values in time, as shown in Fig. 10. The magnitude of the unsteady wall temperature fluctuations for the coarsest mesh (Mesh 1) is shown to be on average about 5 times higher than that of the finest mesh (Mesh 4).

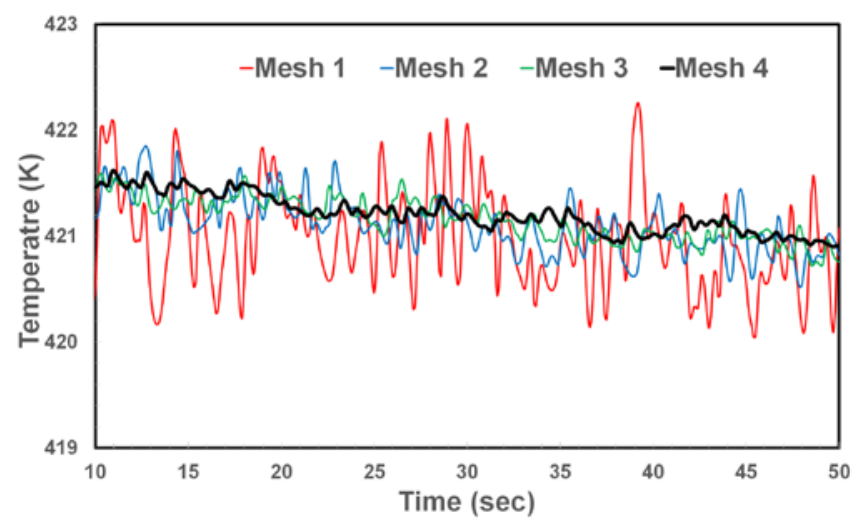

Fig. 10 Time history of interface wall temperature for different Meshes (Direct coupled CHT using LES).

The dependence of the LES CHT case on the solid meshes is in clear contrast to the mesh insensitivity for the same mesh densities in a steady CHT case (Figs.6, 7). This suggests that it is the interplay between the LES and the interface treatment that is responsible for this solid mesh dependency behaviour. 
Note that the differences in the interface wall temperatures between the different meshes are in the order of 1K (Fig.8), which may not appear large. However, we should keep in mind that these LES solutions are obtained at a steady condition (i.e. the isothermal temperature conditions at the inner and outer surfaces, $\mathrm{Ti}=800 \mathrm{~K}$, To $=400 \mathrm{~K}$ ). The situation should effectively correspond to that ane time instant of a transient operation process. The slow conduction of solid means that the thermal field for any given time would be influenced by the accumulated errors from all the past time. Thus, relatively small errors at one time instant may be much more influential in a long transient process than they appear to be at the time instant.

\subsection{Response Analysis for Interface of Directly Coupled CHT}

To help understanding the solid mesh dependency behaviour in a more systematic and insightful manner, we now carry out a simple analysis of the interface treatment for a directly coupled CHT procedure.

A near-wall mesh point in the fluid domain and its counterpart in the solid domain are shown in Fig.11. Once the interior domains on both sides have been updated during a solution, we now need to update the variables at the interface (wall surface) through the interface treatment. Taking a simple one-sided finite difference (FD) to approximate the heat fluxes for both sides, we then have a discrete form of the physical heat flux and temperature continuity across the interface (Eq.5a):

$$
k_{f}\left(\frac{T_{f}-T_{W}}{\Delta x_{f}}\right)=k_{S}\left(\frac{T_{W}-T_{S}}{\Delta x_{S}}\right)
$$

Where $T_{\mathrm{w}}$ is the same wall temperature for both sides and $\mathrm{T}_{\mathrm{f}}$ is fluid temperature at the fluid mesh node and $\mathrm{T}_{\mathrm{s}}$ the temperature at the solid mesh node. $\mathrm{k}_{\mathrm{f}}$, and $\mathrm{ks}_{\mathrm{s}}$ are the thermal conductivities for the two domains respectively. 


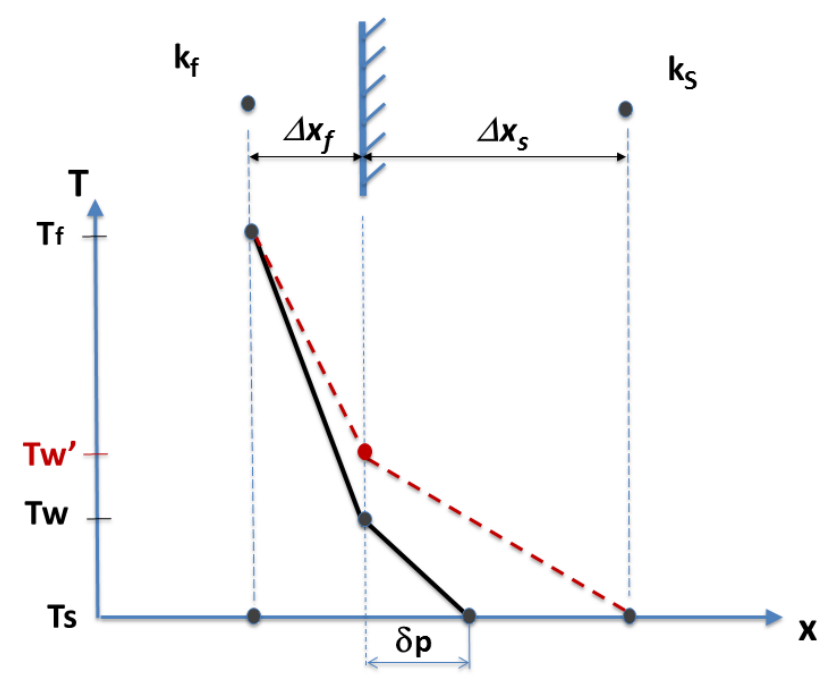

Fig. 11, Temperature variations across fluid-solid interface (Solid line: realistic; Dash line: erroneous).

The wall temperature can thus be updated in terms of the updated fluid and solid temperatures $T_{\mathrm{f}}$ and Ts.

$$
T_{W}=\frac{T_{f} k_{f} / \Delta x_{f}+T_{S} k_{s} / \Delta x_{S}}{k_{f} / \Delta x_{f}+k_{S} / \Delta x_{S}}
$$

Assuming constant thermal conductivities for both sides, we then have a linear relation between the wall temperature and the two temperatures on both sides. An instantaneous temperature can be expressed in terms of a fluctuation about a mean value (e.g. $\left.T_{w}=T_{w 0}+\Delta T_{w}\right)$. The mean temperatures $\left(T_{w 0}, T_{f 0}, T_{s 0}\right)$ are then completely balanced by themselves. Thus, the terms left in Eq.7 will give the relation to balance the temperature fluctuations relative to the corresponding time-mean values.

Given the fluid natural convection as the primary driver, we now examine how the temperature in the solid part will respond to a given incoming fluid unsteady temperature disturbance $\Delta \mathrm{T}_{\mathrm{f}}$.

The key to the accuracy and consistency of the wall temperature response $\Delta \mathrm{T}_{\mathrm{w}}$ is how far the temperature disturbance can propagate in the solid. For a 1D semi-infinite domain, this is marked by the penetration depth (e.g.[18]):

$$
\delta_{P}=\sqrt{8 \alpha \delta t}
$$

The time scale $\delta t$ is given by the time period of the disturbance. Take a typical turbulence disturbance of $1 \mathrm{~Hz}$ (see Figs.4, 5) and the diffusivity $\alpha$ for steel used for the present work. The penetration depth is 
calculated to be $5.7 \mathrm{~mm}$. Clearly if the penetration depth is smaller than the mesh spacing, any FD approximation of the gradient will be erroneous. For the coarsest solid mesh (Mesh 1), the mesh cell spacing of the solid domain is $27.5 \mathrm{~mm}$ (Table 1), which turns out to be more than 4 times larger than the penetration depth for the disturbances at this frequency. The mesh spacing of Mesh 3 is just about to be comparable to the penetration depth. Now we start to see why the solid mesh dependence is much weaker when the mesh is finer than Mesh 3, as indicated in Fig.10.

For simplicity, we assume that the temperature can be reasonably approximated by a linear variation between the wall and the penetrated distance. A correct finite difference approximation for the temperature wall response (indicated as the solid line shown in Fig.11) would be when the mesh spacing is $\delta$ p. Also given $\Delta \mathrm{T}_{\mathrm{s}}=0$, the wall temperature disturbance thus becomes.

$$
\Delta T_{W}=\frac{\Delta T_{f} k_{f} / \Delta x_{f}}{k_{f} / \Delta x_{f}+k_{s} / \delta_{P}}
$$

This can be contrasted to the erroneous temperature response $\left(\mathrm{T}_{\mathrm{w}}{ }_{\mathrm{w}}=\mathrm{T}_{\mathrm{w} 0}+\Delta \mathrm{T}^{\prime}{ }_{\mathrm{w}}\right.$, as shown in Fig.11), calculated based on the mesh spacing $\Delta \mathrm{x}_{\mathrm{s}}$, larger than $\delta$ p.

$$
\Delta T_{W}^{\prime}=\frac{\Delta T_{f} k_{f} / \Delta x_{f}}{k_{f} / \Delta x_{f}+k_{s} / \Delta x_{s}}
$$

Clearly, $\Delta T_{W}$ is smaller than $\Delta T_{W}^{\prime}$. The wall temperature fluctuation is erroneously amplified by a factor of:

$$
\frac{\Delta T_{w}^{\prime}}{\Delta T_{W}}=\frac{1+\left(\frac{k_{s}}{k_{f}}\right)\left(\frac{\Delta x_{f}}{\delta_{P}}\right)}{1+\left(\frac{k_{s}}{k_{f}}\right)\left(\frac{\Delta x_{f}}{\Delta x_{s}}\right)}
$$

Effectively the heat flux continuity for the two sides asks for a complete energy balance between the fluid mesh point and the solid one locally and instantly, which is simply impossible if the disturbance cannot reach the solid mesh point. However, for the given coarse solid mesh, Eq.10 will force the wall temperature fluctuation to rise fictitiously to a higher value, resulting in a somewhat lower gradient (thus a lower heat flux) to still satisfy the temperature and flux continuity instantaneously, as indicated by the dash line in Fig.11. 
Table 2 gives the ratios between the calculated erroneous responses with the first three meshes and the correct value when $\Delta \mathrm{x}_{\mathrm{S}}=\delta_{\mathrm{p}}$. The impact on the magnitude of the wall temperatures is clear. Quite remarkably, the maximum ratio of 4.60 in the temperature fluctuation magnitude estimated by this simple analysis is very much in line with the observed behaviour from the computational results (Fig.10).

Table 2: Mesh Dependence of $T_{w}$ and $q(\mathrm{ks}=16.3 \mathrm{~W} / \mathrm{m} \mathrm{K})$

(predicted by the interface response analysis).

\begin{tabular}{|l|c|c|c|}
\hline Solid Mesh & Mesh 1 & Mesh 2 & Mesh 3 \\
\hline$\Delta T_{W}^{\prime} / \Delta T_{W}$ & $\mathbf{4 . 6 0}$ & $\mathbf{2 . 2 7}$ & $\mathbf{1 . 1 2 6}$ \\
\hline$\Delta q^{\prime} / \Delta q$ & $\mathbf{0 . 9 4 1}$ & $\mathbf{0 . 9 7 9}$ & $\mathbf{0 . 9 9 7}$ \\
\hline
\end{tabular}

We can similarly relate the heat flux perturbation to a given fluid temperature disturbance. Thus, when the penetration depth is equal to the first solid mesh spacing, we should have an expected (correct) heat flux fluctuation:

$$
\Delta q=k_{f} \frac{\Delta T_{f}}{\Delta x_{f}}\left(\frac{k_{s} \Delta x_{f}}{k_{s} \Delta x_{f}+k_{f} \delta_{P}}\right)
$$

This again can be contrasted to the erroneous heat flux response $\Delta q^{\prime}$ calculated based on the mesh spacing $\Delta \mathrm{x}_{\mathrm{S}}$ larger than $\delta_{\mathrm{P}}$.

$$
\frac{\Delta q^{\prime}}{\Delta q}=\frac{1+\left(\frac{k_{f}}{k_{s}}\right)\left(\frac{\delta_{P}}{\Delta x_{f}}\right)}{1+\left(\frac{k_{f}}{k_{s}}\right)\left(\frac{\Delta x_{s}}{\Delta x_{f}}\right)}
$$

Thus, the predicted heat flux fluctuation $\Delta q^{\prime}$ at a coarser mesh would be smaller than the target value $\Delta \mathrm{q}$. For the present cases, the ratios between the erroneous flux fluctuations and the correct one with different solid meshes are also included in Table 2. The errors in the flux fluctuations are relatively much smaller than those for the wall temperatures, as expected given the relative small magnitudes of the wall temperature fluctuations. The heat flux results of the analysis shown in Table 2 are also in line with the 
present computational results. In general, the analysis suggests that a direct CHT interface treatment would tend to over-predict wall temperature fluctuations and under-predict heat flux fluctuations.

It should be noted that the present interface response analysis, though simple, should be applicable for other LES/DNS based CHT procedures. The interface behaviour may vary depending on the solid properties (e.g. thermal conductivity) as well as the fluid mesh. It is noted that in a previous LES based CHT analysis for a forced convection around a turbine blade with a Thermal Barrier Coating (TBC), a direct coupled CHT solution led to a significant under-prediction (up to 50\%) of heat fluxes as observed by He [9]. Given a typical low conductivity of TBC, it is thus of interest to get some indications of the sensitivity of unsteady wall temperature and flux responses to the solid conductivity.

For simplicity and direct comparability, we simply reduce the solid thermal conductivity by a factor of $10\left(\mathrm{ks}_{\mathrm{S}}=1.63 \mathrm{~W} / \mathrm{m} \mathrm{K}\right)$, whist keeping other parameters the same. This low value of thermal conductivity is in the range of those of TBC. The corresponding $\mathrm{T}_{\mathrm{w}}$ and $\mathrm{q}$ fluctuations for this low solid conductivity as predicted by the interface response are listed in Table 3. Clearly, we now have a much stronger mesh dependence. For the coarsest mesh, the temperature fluctuations may be over-predicted by more than 9 times with the heat fluxes under-predicted by more than $40 \%$. Even the finest mesh (Mesh 4), which is shown to be completely adequate for the case with the nominal thermal conductivity, would not be sufficient for this case with the low solid thermal conductivity. The contrasting interface behaviour between the nominal and low conductivities of the solid domain also seems to explain the markedly underpredicted heat flux fluctuations, as previously observed [9].

Table 3: Mesh Dependence of $T_{w}$ and $q(k s=1.63 \mathrm{~W} / \mathrm{m} \mathrm{K})$

(predicted by the interface response analysis).

\begin{tabular}{|l|c|c|c|c|}
\hline Solid Mesh & Mesh 1 & Mesh 2 & Mesh 3 & Mesh 4 \\
\hline$\Delta T_{W}^{\prime} / \Delta T_{W}$ & $\mathbf{9 . 0 5}$ & $\mathbf{5 . 5 9}$ & $\mathbf{3 . 1 6}$ & $\mathbf{1 . 7 0}$ \\
\hline$\Delta q^{\prime} / \Delta q$ & $\mathbf{0 . 5 8 6}$ & $\mathbf{0 . 7 1 4}$ & $\mathbf{0 . 8 8 9}$ & $\mathbf{0 . 9 6 4}$ \\
\hline
\end{tabular}




\section{LOOSELY COUPLED CHT METHOD}

The results of the sensitivity studies and the response analysis for an interface above provide a clear motivation for seeking alternatives to the direct coupling interface treatment as it stands. Simply put, the current direct interface treatment is unable to pass unsteady flow disturbances above certain frequencies. This extra mesh sensitivity to the solid mesh is rather undesirable to say at least, given the range of the high frequency turbulence disturbances which the high-fidelity flow solvers (LES in this case) are able and meant to resolve. One simple option to consider would be to separate the interface from these highfrequency disturbances. A consistent way to do this is a loosely coupled approach, where the interface treatment will only 'see' an averaged parameter from the fluid side.

For a transient turbine operation of practical interest, in addition to the inherent time scale disparity between fluid and solid domains, we also face the time scale disparity between turbulence movement (in sub-seconds) and the practical operational process (in hours/days). These disparities can be effectively dealt with by a multi-scale time integration approach (He and Fadl [19]), which has been implemented in FLUENT, validated and demonstrated for forced convection problems. The multi-scale loosely coupled method will now be adopted and applied to transient natural convection. The main elements will be briefly described here.

\subsection{Flow Variable and Time-Scale Decomposition}

A flow decomposition is taken primarily in order to better deal with the time scale disparity. In addition, the decomposition is introduced to enable accommodating eddy-resolved LES solvers for turbulence.

Keep in mind that we have to deal with two distinctive ranges of time scales: the long-time scales for the solid (and a slow operational process) and the short ones for resolving turbulence fluctuations. A decomposition for the fluid part will be made accordingly. A general flow variable can be expressed as:

$$
U(x, t)=\tilde{U}(x, t)+U^{\prime}(x, t)
$$

where $\widetilde{U}$ is the flow variable after a low-pass filtering so that it only contains long wavelength disturbances comparable to the large time scales for the solid domain.

The realization of the above flow variable decomposition is accompanied by a corresponding discrete time step decomposition. For any sampling point in a discrete time domain, the time instant $\mathrm{t}$ is marked by a large-scale time instant $t_{\mathrm{M}}$ corresponding to a solid domain solution, and a small-scale time instant $t_{M i}$ relative to $t_{M}$, 


$$
\mathrm{t}=\mathrm{t}_{\mathrm{M}}+\mathrm{t}_{\mathrm{Mi}} \quad(\mathrm{i}=1,2, \ldots \mathrm{m})
$$

The filtering is effectively a time-averaging of small scale (turbulence) disturbances over m local time steps ('LES fluid steps'),

$$
\tilde{U}\left(t_{M}\right)=\frac{1}{m} \sum_{i=1}^{m} U\left(t_{M i}\right)
$$

where $\mathrm{m}$ is a sufficiently large number for a local time-averaging to be adequately obtained. Given the definition of time-filtering, it then follows, $\widetilde{U^{\prime}}=0$.

\subsection{Two-Scale Integrations of Flow Equations}

The unsteady flow equation after the low-pass filtering becomes:

$$
\frac{\partial \widetilde{U}}{\partial t}+\widetilde{R_{f}(U)}=0
$$

Note that $\widetilde{R_{f}(U)} \neq R_{f}(\widetilde{U})$ due to nonlinearity of advection terms in the flow equations.

In order to satisfy the above equation at a long solid time instant $t_{\mathrm{M}}$, noting that $\partial \widetilde{U^{\prime}} / \partial t=0$, we rewrite the above equation as:

$$
\left(\frac{\partial \tilde{U}}{\partial t}\right)_{M}+\frac{1}{m} \sum_{i=1}^{m}\left[\frac{\partial U^{\prime}}{\partial t}+R_{f}(U)\right]_{i}=0
$$

Given that a filtered flow variable does not change with the local short fluid time scale for a given solid time instant $t_{M}$, the corresponding time-gradient of the filtered flow variable $\left(\frac{\partial \tilde{U}}{\partial t}\right)_{M}$ can thus be treated as a constant source term. As such, the above equation (Eq.18) will hold if the following equations hold at m local time steps respectively:

$$
\left(\frac{\partial \tilde{U}}{\partial t}\right)_{M}+\left[\frac{\partial U^{\prime}}{\partial t}+R_{f}(U)\right]_{i}=0 \quad(\text { for } \mathrm{i}=1,2, \ldots \mathrm{m})
$$

Similarly, at this given solid time instant, the filtered part of the flow variable $\tilde{U}$ should be regarded as being frozen when integrating in the local fluid time. We thus have:

$$
\left.\frac{\partial U^{\prime}}{\partial t}=\frac{\partial(U-\tilde{U})}{\partial t}=\frac{\partial U}{\partial t} \quad \text { (for local time } \mathrm{t}=\mathrm{t}_{\mathrm{M} 1}, \mathrm{t}_{\mathrm{Mm}}\right)
$$


Based on the above reasoning, the time-domain unsteady flow equation to be solved locally at the given solid time step $\mathrm{M}$ becomes:

$$
\left.\left[\frac{\partial U}{\partial t}+R_{f}(U)\right]_{i}=-\left(\frac{\partial \tilde{U}}{\partial t}\right)_{M} \quad \text { (for } \mathrm{i}=1,2, . ., \mathrm{m}\right)
$$

\subsection{Fluid-Solid Equation System}

Using a time-marching solver by introducing a pseudo time $\tau$, we now have a triple-timing form of the unsteady flow equations.

$$
\frac{\partial U}{\partial \tau}+R_{f}(U)=-\left(\frac{\partial \tilde{U}}{\partial t}\right)_{M}-\left(\frac{\partial U}{\partial t}\right)_{m}
$$

where subscripts ' $M$ ' and ' $m$ ' denote the solid (long) and fluid (short) time scales respectively. In terms of the interactions between the two scales, the large scale physical time-gradient provides a base-value contribution to balancing the unsteady flow equations at each small time-step, whilst the averaged small scale unsteady flow disturbances collectively affect those of the large scales.

For the solid domain on the other hand, the dual timing formulation remains as the large time step should be indeed determined by the required solid temporal resolution.

$$
\left(\frac{\partial \tilde{T}}{\partial \tau}\right)+R_{s}(\tilde{T})=-\left(\frac{\partial \tilde{T}}{\partial t}\right)_{M}
$$

All these extra source terms are added to the flow equations of the baseline flow solver and that of the baseline solid conduction solver through the user defined function (UDF) in FLUENT.

\subsection{Fluid-Solid Interface for Loosely Coupled CHT}

In a loosely coupled CHT procedure, the fluid and solid domains are solved separately in sequence. Each consecutive pair of solutions for the two domains can be called a 'CHT coupling cycle'. The interfacing conditions for a loosely coupled CHT are effectively the boundary conditions for each domain, updated once in each coupling cycle. The key feature relevant to the present LES based loosely coupled CHT procedure is that the interface will now only 'see' the locally time-averaged ('filtered') fluid side for any given solid time step $\mathrm{t}_{\mathrm{M}}$ during a large scale transient process.

The baseline boundary conditions for the loosely coupled CHT directly follow the common physical conditions of temperature and heat flux continuity across the interface [17]. A stable interface treatment 
can be achieved by simply specifying a wall temperature condition for the fluid domain and specifying a heat flux for the solid domain iteratively as analysed by Giles [20]. This simple Dirichlet (fluid) and Neumann (solid) interface treatment can be easily implemented as (marching from coupling cycle $\mathrm{n}$ to $n+1)$ :

$$
\begin{aligned}
& \left(\widetilde{T_{f}}\right)_{n+1}=\left(\widetilde{T_{S}}\right)_{n} \\
& \left(\widetilde{q_{S}}\right)_{n+1}=\left(\widetilde{q_{f}}\right)_{n+1}
\end{aligned}
$$

Clearly, when the coupling cycles converge, the above will be a direct manifestation of the physical temperature and flux continuity across the interface. There is also an option for an accelerated hybrid interface treatment as described by He and Fadl [19].

\section{RESULTS OF THE LOOSELY COUPLED METHOD}

The following examples computed using the present multi-scale loosely coupled CHT procedure are included here to illustrate two main points of the present interest:

a. The present loosely coupled solutions should improve the computational consistency with much reduced sensitivity and dependence on the solid mesh.

b. The present multi-scale transient CHT procedure can provide an improvement in computational accuracy over the quasi-steady model commonly adopted in previous loosely coupled CHT methods.

The same test case as presented in section 2.3 is now examined by the present loosely coupled CHT procedure, with the four different meshes, as given in Table 1 . The time-averaged interface temperatures for the two extreme mesh densities tested are compared as shown in Fig.12. The difference between the two solutions is also shown in the figure. It can be seen that the two solutions of the two meshes are in good agreement. The differences between the two are now much smaller than those for the corresponding direct coupled LES solutions (Fig.8). The much reduced mesh dependency for the present loosely coupled solutions is now largely comparable to that of the steady CHT solution (Fig. 6). 


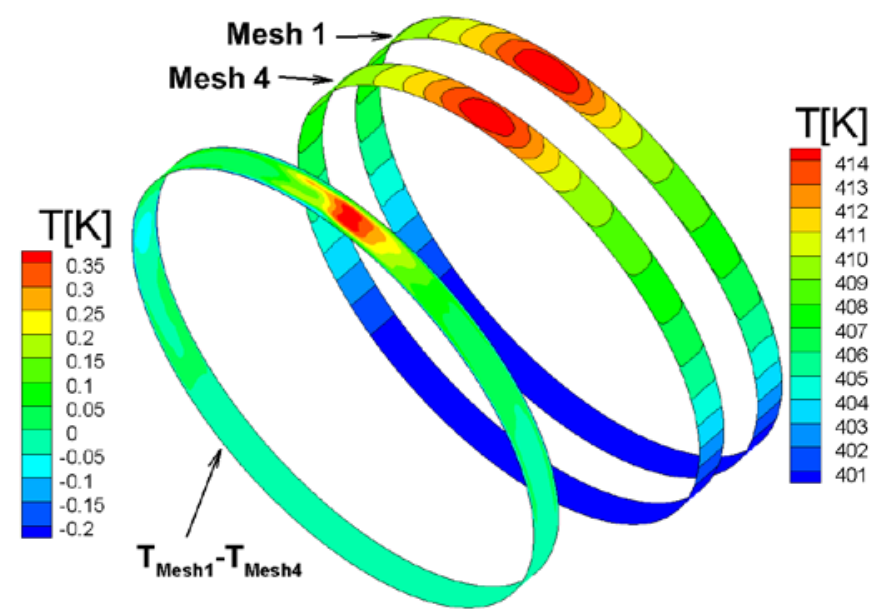

Fig. 12 Interface temperatures for different meshes (Loosely coupled CHT using LES).

The time-averaged heat fluxes computed by the present loosely coupled procedure for the maximum and minimum mesh densities tested are shown in Fig.13. The maximum difference in the heat flux is now only about 4\%, which is about 4 times smaller than that of the direct coupled CHT solutions (Fig.9). Thus, we now have a marked improvement in the mesh-dependence on the solid domain mesh for the present loosely coupled LES based CHT procedure, compared to its directly coupled CHT counterpart.

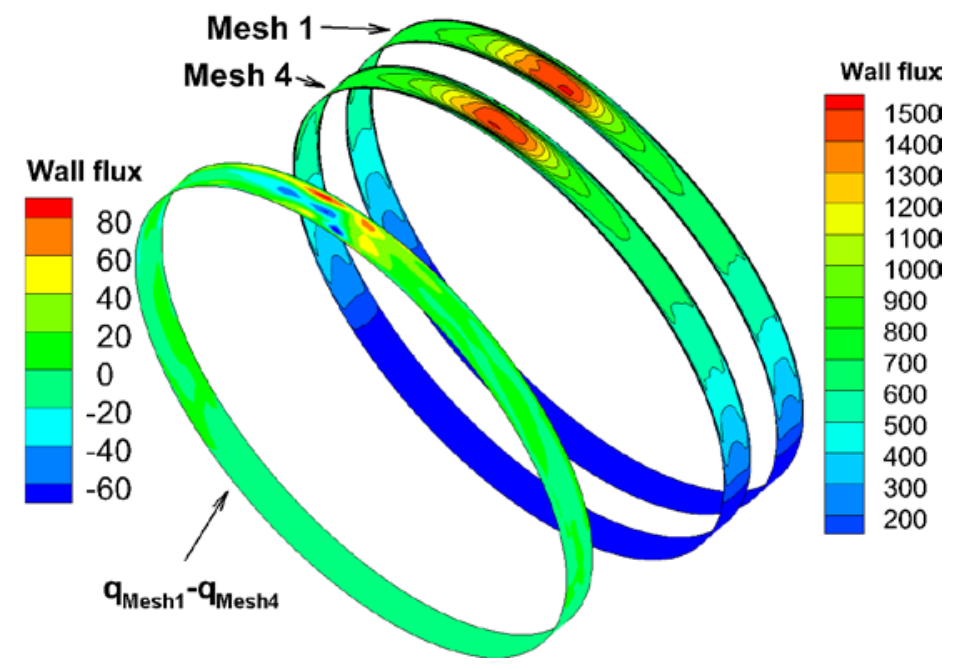

Fig. 13 Interface time-averaged heat flux for different meshes (Loosely coupled CHT using LES).

We use two further examples to illustrate the levels of differences between a quasi-steady model and the present triple-timing unsteady model for a transient process. The transient process is generated by a linear variation of $100 \mathrm{~K}$ in the fluid domain inner wall temperature for a given time period. Thus, the temporal gradient of the process is given by the length of the time period over which the linear variation takes place. We will compare the time-averaged heat fluxes at the fluid-solid interface between the present 
unsteady loosely coupled procedure and the quasi-steady mode of the same procedure with the unsteady source term being simply switched off.

It is worth noting that in terms of the methodology itself, the quasi-steady mode of the present procedure is comparable to other previous LES based loosely coupled methods, though these previous methods have been so far only applied to forced convection. We need then stress that for the present procedure, running it in a quasi-steady mode or in an unsteady triple-timing mode would consume largely the same amount of computing time. In other words, the present triple-timing loosely coupled method is almost as efficient as those previous loosely coupled CHT methods, but we do not need to make use of the quasi-steady assumption.

First, we consider a very fast unsteady process that takes 1 second, i.e. the temporal gradient of the boundary temperature is $100 \mathrm{~K} / 1 \mathrm{~s}$. The time-averaged heat flux distribution at the interface computed by the present loosely coupled method is compared to that by the quasi-steady model, as shown in Fig.14. The difference between the two solutions is also included. The maximum difference due to the quasisteady assumption is as high as $45 \%$.

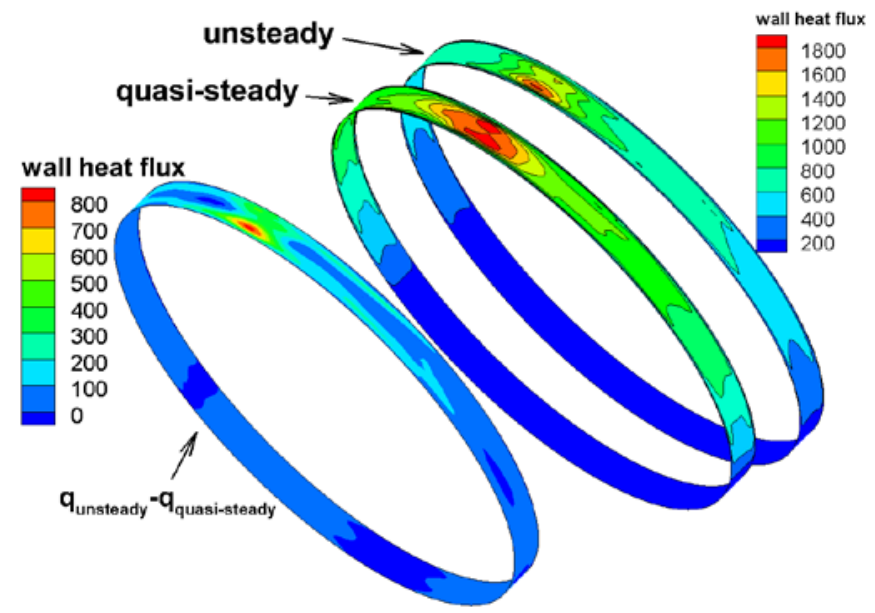

Fig. 14 Time-averaged heat fluxes for transient, 100K /1s (Unsteady flow model vs Quasi-steady flow model).

For a slow process of 30 seconds with a lower gradient, 100K/30s, the difference between the unsteady solution and the quasi-steady one becomes smaller, as shown in Fig.15. Even in this case, the maximum difference is still in the range of about 25\%, which is certainly not negligible.

It should be commented that we have so far only examined the cases with a relatively short period (in seconds) in time. The observed differences in interface temperatures and heat fluxes need to be considered 
in the context of what may happen in a much longer turbine flexible operation process (in hours/days). The thermal field of a solid domain for any given time instant would be influenced by the accumulated effects from all the past time. Thus, small errors at each time instant can collectively have a large impact in a long transient process.

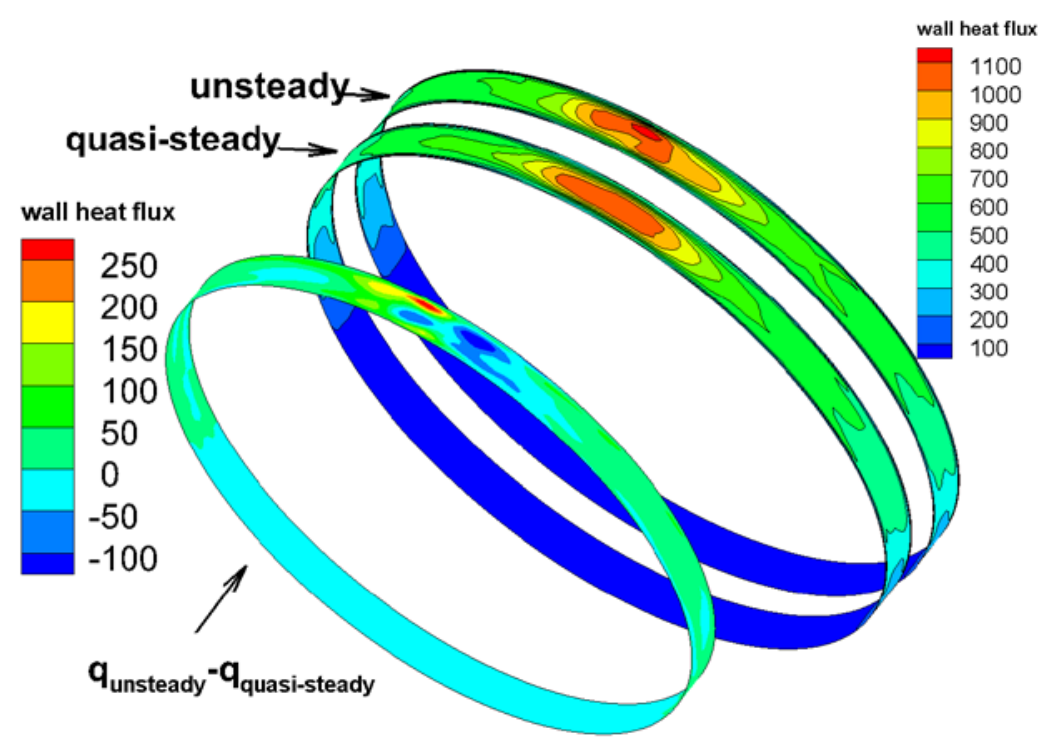

Fig. 15 Time-averaged heat fluxes for transient, 100K /30s

(Unsteady flow model vs Quasi-steady flow model)

\section{CONCLUDING REMARKS}

When a fluid domain LES solution is directly coupled with a solid domain conduction solution for conjugate heat transfer simulations, an extra mesh dependence on the solid domain mesh may arise. This behaviour has been demonstrated in the present computational studies for natural convection. The observed characteristics are underlined with some further insights and quantifications by a simple response analysis of interface temperatures and fluxes. The present results indicate that a directly coupled LES-CHT solution would tend to over-predict wall temperature fluctuations and under-predict heat flux fluctuations when the solid mesh is not fine enough for high frequency disturbances. The issue is addressed here with a loosely coupled procedure based on a new multi-scale methodology, resulting in a first of the kind LES based CHT application to transient natural convection. To accurately and effectively deal with problems with large time scale disparity, a multi-scale flow decomposition associated with a 
corresponding time-step split is adopted. The resultant triple-timing formulation of the flow equations can be solved efficiently for the fluid-solid loosely coupled system. The method descriptions are presented with case studies and discussions to underpin the problem statement and the understanding of relevant causal links, and to demonstrate the validity and effectiveness of the present methodology and implemented procedure.

\section{ACKNOWLEDGMENTS}

The present work is sponsored by UK Engineering and Physical Science Research Council (EPSRC) and GE Power. The authors would also like to thank Drs Peter Stein and Gabriel Marinescu (GE Power) for their valuable support and interactions.

\section{REFERENCES}

[1] Marinescu, G., Mohr, W. F., Ehrsam, A., Ruffino, P., and Sell, M., 2013, “Experimental Investigation into Thermal Behavior of Steam Turbine Components-Temperature Measurements with Optical Probes and Natural Cooling Analysis,” J. Eng. Gas Turbines Power, 136(2), p. 21602.

[2] Marinescu, G., Stein, P., and Sell, M., 2015, "Natural Cooling and Startup of Steam Turbines: Validity of the Over-Conductivity Function,” J. Eng. Gas Turbines Power, 137(11), p. 112601.

[3] Maffulli, R., and He, L., 2014, "Wall Temperature Effects on Heat Transfer Coefficient for High-Pressure Turbines,” J. Propuls. Power, 30(4), pp. 1080-1090.

[4] Zhang, Q., and He, L., 2014, "Impact of Wall Temperature on Turbine Blade Tip Aero-Thermal Performance,” J. Eng. Gas Turb. Power, Vol.136, No.5, 052602.

[5] Maffulli, R., and He, L., 2017, "Impact of Wall Temperature on Heat Transfer Coefficient and Aerodynamics for Three-Dimensional Turbine Blade Passage,” J. Therm. Sci. Eng. Appl., 9(4), p. 41002.

[6] Topel, M., Genrup, M., Jöcker, M., Spelling, J., and Laumert, B., 2015, “Operational Improvements for Start-Up Time Reduction in Solar Steam Turbines,” ASME J. Eng. Gas Turb. Power, 137(4), pp. 042604042604, 2015.

[7] Born, D., Stein, P., Marinescu, G., Koch, S., and Schumacher, D., 2016, "Thermal Modelling of an Intermediate Pressure Steam Turbine by Means of Conjugate Heat Transfer: Simulation and Validation,” ASME J. Eng. Gas Turb. Power, 139(3), pp. 031903-031903, 2016. 
[8] He, L., and Oldfield, M. L. G., 2011, “Unsteady Conjugate Heat Transfer Modeling,” J. Turbomach., 133(3), p. 31022.

[9] He, L., 2013, “Fourier Spectral Modelling for Multi-Scale Aero-Thermal Analysis,” Int. J. Comut. Fluid Dyn., 27(2), pp. 118-129.

[10] Shahi, M., Kok, J. B. W., Roman Casado, J. C., and Pozarlik, A. K., 2014, "Study of Unsteady Heat Transfer as a Key Parameter to Characterize Limit Cycle of High Amplitude Pressure Oscillations," ASME Paper GT2014-26311, 2014.

[11] Sun, Z., Chew, J. W., Hills, N. J., Volkov, K. N., and Barnes, C. J., 2010, "Efficient Finite Element Analysis/Computational Fluid Dynamics Thermal Coupling for Engineering Applications,” J. Turbomach., 132(3), p. 31016.

[12] Wang, Z., Corral, R., Chaquet, J. M., and Pastor, G., 2013, “Analysis and Improvement of a Loosely Coupled Fluid-Solid Heat Transfer Method,” ASME Paper GT2013-94332.

[13]Duchaine, F., Corpron, A., Pons, L., Moureau, V., Nicoud, F., and Poinsot, T., 2009, "Development and Assessment of a Coupled Strategy for Conjugate Heat Transfer with Large Eddy Simulation: Application to a Cooled Turbine Blade,” Int. J. Heat Fluid Flow, 30(6), pp. 1129-1141.

[14] Duchaine, F., Boileau, M., Sommerer, Y., and Poinsot, T., 2014, "Large Eddy Simulation of Flow and Heat Transfer Around Two Square Cylinders in a Tandem Arrangement,” J. Heat Transfer, 136(10), p. 101702.

[15] Errera, M. P., and Baque, B., 2013, “A Quasi-Dynamic Procedure for Coupled Thermal Simulations,” Int. J. Numer. Methods Fluids, 72(11), pp. 1183-1206.

[16] Fadl, M., He L., Stein, P., and Marinescu, G., 2017, “Assessment of Unsteadiness Modelling for Transient Natural Convection,” ASME Paper GT2017-53592, in press J. Eng. Gas Turbines Power.

[17]Perelman, T. L., 1961, “On Conjugated Problems of Heat Transfer,” Int. J. Heat Mass Transf., 3(4), pp. 293-303.

[18]Faghri, A., and Zhang, Y., 2010, “Advanced Heat and Mass Transfer,” Global Digital Press, Columbia, MO, p. 934.

[19]He, L., and Fadl, M., 2017, “Multi-Scale Time Integration for Transient Conjugate Heat Transfer,” Int. J. Numer. Methods Fluids, 83(12), pp. 887-904.

[20]Giles, M. B., 1997, "Stability Analysis of Numerical Interface Conditions in Fluid-Structure Thermal Analysis,” Int. J. Numer. Methods Fluids, 25(4), pp. 421-436. 